NASA Technical Memorandum 103862

20605

8.24

\title{
Analysis of the Intel 386 and i486 Microprocessors for the Space Station Freedom Data Management System
}

\section{Yuan-Kwei Liu}

(NASA-TM-103862) ANALYSIS OF THE INTEL 386 AND I486 MICKOPROCESSORS FOR THE SPACE

STATION FREEDOM DATA MANAGEMENT SYSTEM (NASA) 24 P
CSCL 098
$N 71-25687$

Unclas

Unclas

May 1991

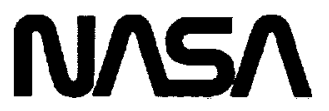

National Aeronautics and

Space Administration 


\section{Analysis of the Intel 386 and i486 Microprocessors for the Space Station Freedom Data Management System}

Yuan-Kwei Liu, Ames Research Center, Moffett Field, California

May 1991

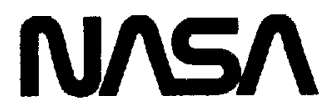

National Aeronautics and

Space Administration 


\section{SUMMARY}

This report analyzes the feasibility of upgrading the Intel $386^{a}$ microprocessor, which has been proposed as the baseline processor for the Space Station Freedom (SSF) Data Management System (DMS), to the more advanced $1486^{\mathrm{a}}$ microprocessor. It is part of an effort funded by the National Aeronautics and Space Administration (NASA) Space Station Freedom Advanced Development program.

The items compared between the two processors include the instruction set architecture, power consumption, the MIL-STD-883C Class S (space) qualification schedule, and performance.

The advantages of the i486 over the 386 are lower power consumption and higher floatingpoint performance in speed. The i 486 on-chip cache, however, has neither parity check nor error detection and correction circuitry. In space, the probability of having the contents of the cache altered, is higher than on the ground due to the higher radiation exposure. Therefore, it is necessary to measure the performance of the i 486 with the cache disabled.

The i486 with on-chip cache disabled, however, has lower integer performance in speed than the 386 without cache, which is the current DMS design choice. Using external cache with a specially designed cache controller can improve the performance of the i486, but the added complexity may not provide a better solution than adding cache to the 386 .

The benchmark performance of a 386-based prototype Flight Equivalent Unit (FEU), which is the closet configuration to the DMS design as of April 1991, is only about $50 \%$ of a PS/2 Model 70 with cache, which is generally considered as a 4 MIPS (million instructions per second) computer. Adding cache to the $386 / 387 \mathrm{DX}$ memory hierarchy appears to be the most beneficial way to enhance computation-intensive performance for the current DMS design at this time.

a 386,387 and 1486 are trademarks of Intel Corporation. 


\section{OVERVIEW OF INTEL 386 AND i486 MICROPROCESSORS}

\subsection{Intel 386 Microprocessor}

Intel's 80xxx family of microprocessors was initiated with the 16-bit 8086 processor in 1978. Intel then developed the 8088 which has a 16-bit internal architecture with an 8-bit data bus interface. The 8088 was chosen by IBM (International Business Machines Corporation) for use in the IBM PC (personal computer) in 1982. The 8087 floating-point coprocessor adds arithmetic, trigonometric, exponential, and logarithmic instructions to the $8086,8088,80186$, and 80188 instruction set. The $80 \mathrm{xxx}$ instruction set architecture (ISA) is upward compatible as each new ISA remains a super set of the previous ISA as more instructions are added. Figure 1 shows the relationship among these processors.

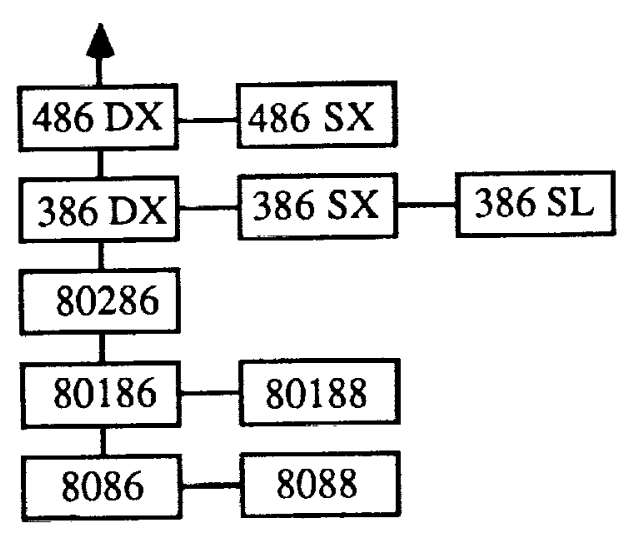

Central Processors

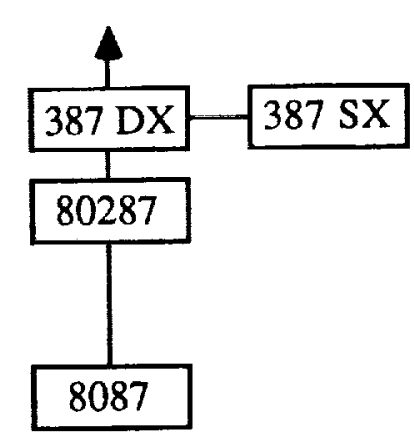

Floating-Point Coprocessors

FIGURE 1. The Intel 80xxx Microprocessor Family Tree

The 386 family of microprocessors includes the $386 \mathrm{DX}, 386 \mathrm{SX}$, and $386 \mathrm{SL}$ processors. The $386 \mathrm{DX}$ is a full 32-bit processor. The $386 \mathrm{SX}$ and SL have 32-bit internal architectures with a 16-bit data bus interface. The $386 \mathrm{DX}$ and $387 \mathrm{DX}$ (floating-point coprocessor) are the baseline embedded processors for the standard data processor (SDP) of the Space Station Freedom (SSF) Data Management System (DMS), Electrical Power System (EPS), and other systems. The 386 SX and 387 SX are baselined for the multiplexer-demultiplexer (MDM) of the SSF. In this analysis only the $386 \mathrm{DX}$ and $387 \mathrm{DX}$ were used.

The 386 DX has eight general-purpose 32-bit registers. The instruction set offers 8-, 16-, and 32-bit data types (ref. 1). It addresses 4 gigabytes $\left(2^{32}\right)$ of memory and has an on-chip memory management unit (MMU) that supports virtual memory management. The commercial $386 \mathrm{DX}$ processors are available at clock rates of 20,25 , and $33 \mathrm{MHz}$ as of April 1991. The corresponding internal bus bandwidths are 40,50 , and 66 megabytes per second.

The 386 DX has three modes of operation: (1) virtual 8086 mode, which enables the processor to multi-task standard DOS (Disk Operating System) applications; (2) real address mode 
(real mode), in which the 386 DX behaves like an $8088 / 8086$, with the original 640 -Kbyte/1Mbyte limitations; and (3) protected virtual address mode (protected mode), in which the $386 \mathrm{DX}$ can execute multiple programs concurrently with each program being protected (ref. 2 ). The protected mode utilizes the full capacities of the $386 \mathrm{DX}$, such as the virtual memory addressing and multitasking, which allows multiple programs to execute concurrently.

There are four privilege levels in the protected mode, from level 0 through 3 . These privilege levels are discussed in detail in Section 4.3.

The $387 \mathrm{DX}$ floating-point coprocessor is designed to work with the $386 \mathrm{DX}$. The $387 \mathrm{DX}$ is compliant with the ANSI/IEEE 754-1985 floating-point standard. It expands the $386 \mathrm{DX}$ data types to include 32-, 64-, and 80-bit floating-point, and 32-, and 64-bit integers. The current DMS design uses the $387 \mathrm{DX}$ coprocessor.

\subsection{Intel i486 Microprocessor}

The i486 is a full 32-bit microprocessor which is currently the top-of-the-line processor in the Intel $80 \mathrm{xxx}$ family. The on-chip integration of the $\mathrm{i} 486$ includes an 8 -Kbyte cache, a floating-point unit (FPU), and a paged, virtual memory management unit (ref. 3). The i486 supports multiprocessor instructions, cache consistency protocols, second level cache, and other multiprocessor support hooks. The i486 ISA is upward compatible with the 386/387 DX ISA with six more instructions added. The commercial i 486 chips are available at clock rates of 25 and 33 MHz as of April 1991.

The i486's on-chip cache uses a write-through memory update policy, i.e., the information is written to both the cache and the main memory. The advantages of this (as compared to writeback) are that main memory is always up to date and the memory logic design is simpler. The disadvantage of the policy is increased bus traffic. The on-chip cache is fundamental to achieving

the higher performance level of the $80 \times x x$ family and is discussed in detail in Sections 4.1. and 4.2 .

Although the 8-Kbyte cache is considerably smaller than the 64 - and 128-Kbyte external caches built into many 386-based PCs, it is considerably more sophisticated. Because of the small cache size and write-through memory policy, Intel has introduced external second-level caches to improve the $i 486$ performance. 


\section{COMPUTER CONFIGURATION FOR THE COMPARISON}

\subsection{Hardware Configuration}

The hardware configuration used for this comparison included a commercial IBM PS/2b Model 70-A21 computer, which has an Intel $386 \mathrm{DX}$ processor. An Intel $387 \mathrm{DX}$ floating-point coprocessor was added to the computer. The clock rate of the computer was $25 \mathrm{MHz}$. The PS/2 comes with 64-kilobyte (KB) of cache memory and 2-megabyte (MB) of main memory. An additional $2 \mathrm{MB}$ of main memory was added to the PS/2. The proposed DMS design includes 20$\mathrm{MHz} 386 \mathrm{DX}$ and $387 \mathrm{DX}$ processors and $4 \mathrm{MB}$ of main memory. The cache is not included in the current DMS design. A summary of the PS 2 and other configurations are shown in Table 1.

TABLE 1. Summary of configurations

\begin{tabular}{|l|l|l|l|l|}
\hline Items & $\begin{array}{l}\text { PS/2 } \\
\text { Model 70 }\end{array}$ & i486 & $\begin{array}{l}\text { Prototype } \\
\text { EDP }\end{array}$ & $\begin{array}{l}\text { Prototype } \\
\text { FEU }\end{array}$ \\
\hline Microprocessors & 386 and 387 DX & i486 & 386 and 387 DX & 386 and 387 DX \\
\hline Clock & $25 \mathrm{MHz}$ & $25 \mathrm{MHz}$ & $20 \mathrm{MHz}$ & $20 \mathrm{MHz}$ \\
\hline External cache & $64 \mathrm{~KB}$ & $\mathrm{~N} / \mathrm{A}$ & $\mathrm{N} / \mathrm{A}$ & $\mathrm{N} / \mathrm{A}$ \\
\hline Internal cache & $\mathrm{N} / \mathrm{A}$ & $8 \mathrm{~KB}$ & $\mathrm{~N} / \mathrm{A}$ & $\mathrm{N} / \mathrm{A}$ \\
\hline Main memory & $4 \mathrm{MB}$ & $4 \mathrm{MB}$ & $4 \mathrm{MB}$ & $4 \mathrm{MB}$ \\
\hline $\begin{array}{l}\text { Error correction } \\
\text { codes }\end{array}$ & N/A & N/A & N/A & Yes \\
\hline $\begin{array}{l}\text { Single event } \\
\text { upset scrub }\end{array}$ & N/A & N/A & N/A & Yes \\
\hline Operating system & LynxOS (v 1.2) & LynxOS (v 1.2) & LynxOS (v 2.0) & LynxOS (v 2.0) \\
\hline Compiler & Lynx C & Lynx C & Lynx C & Lynx C \\
\hline Benchmark 1 & Dhrystone & Dhrystone & Dhrystone & Dhrystone \\
\hline Benchmark 2 & Whetstone & Whetstone & Whetstone & Whetstone \\
\hline
\end{tabular}

As of April 1991, the Model 70-A21 was the only PS/2 model that can be upgraded to the i486. To upgrade the processor, an IBM PS/2 486/25 Power Platform (a board containing a 25$\mathrm{MHz}$ i486 processor), was used to swap with the $386 / 387$ DX board.

In addition to the commercial PS/ 2 computer, two additional configurations which are closer to the DMS flight design were also used for the performance comparison: a prototype EDP

b $\mathrm{PS} / 2$ is a registered trademark of International Business Machine Corporation. 
(Embedded Data processor) and an prototype FEU (Flight Equivalent Unit). The configuration of the prototype EDP is similar to the PS/2 Model 70 except for the clock speed and the external cache. The prototype FEU is the closet configuration to the DMS design as of April 1991: it has no cache memory, but it has ECC (Error Correction Codes) and single event upset scrub for radiation tolerance in its main memory.

\subsection{Software Configuration}

LynxOS $^{c}$ is a Unix ${ }^{d}$-based real-time operating system. LynxOS, which includes Lynx realtime operating system kernel and device drivers, has been selected to be used in the DMS. Version 1.2 of LynxOS was used on the PS/2 and version 2.0 was used on the prototype FEU and the prototype EDP.

The features of LynxOS include (1) deterministic response; (2) pre-emptive kernel; (3) IEEE (Institute of Electrical and Electronics Engineers) POSIX (Portable Operating System Interface for Computer Environments) P1003.1 compliance; and (4) contiguous files (ref. 4).

\subsection{Benchmark Programs}

Benchmark programs are used to measure the performance of a processor and the efficiency of a compiler. The $\mathrm{C}$ version of the Dhrystone (version 2.1) and Whetstone (version 1.0) benchmark programs were used for this performance comparison. These two benchmarks are synthetic programs designed to match the average frequency of an operation and operands of a large set of programs. The Lynx C compiler, which has no optimization option as of April, 1991, was used to compile the benchmark programs.

The Dhrystone benchmark, which has no floating-point arithmetic operations, is designed to measure integer performance. The benchmark recommends executing 30,000 cycles on sixteen bit machines, and many more cycles on faster machines. 100,000 cycles were executed for this comparison. The results are measured in "Dhrystones per Second," with higher numbers representing higher performance level.

The Whetstone benchmark is designed to measure a mix of operations typical of scientific computation. The number of cycles can be set before compilation. In this comparison, 100 cycles were executed, which translates to 10 million Whetstone instructions. Elapsed time is used to calculate the results, which are measured in KWIPS (Kilo-Whetstone Instructions Per Second):

\footnotetext{
${ }^{c}$ LynxOS is a registered trademark of Lynx Real-Time Systems Inc.

d Unix is a trademark of AT\&T Bell Laboratories.
} 


$$
\begin{aligned}
\text { Whetstone Performance (KWIPS) } & =\left(10^{*} 10^{6} / \text { Elapsed Time in seconds }\right) / 10^{3} \\
& =10^{4} / \text { Elapsed Time }
\end{aligned}
$$

Again, higher numbers denote higher performance levels. The Dhrystone and Whetstone benchmark results are given in Section 5.4. and examples of the results are shown in Appendix 7.4 . 


\section{THE i486 ON-CHIP CACHE}

\subsection{The i486 On-Chip Cache Organization}

The $\mathbf{i} 486$ has an $8 \mathrm{Kbyte}$ on-chip cache. The cache is a unified (or mixed) cache, i.e. it can contain either instructions or data. The write strategy of the cache is a write-through policy. If the write was a cache hit, the information is written to both the internal cache and external memory. A write to an address not contained in the internal cache will only be written to external memory.

The cache organization is 4-way set associative, i.e. there are 4 blocks (lines) in a set (ref. 3). A block is first mapped onto a set, and then placed anywhere within the set. Each block size is 16 bytes. The $8 \mathrm{Kbyte}$ cache is physically split into four 2-Kbyte caches, each containing 128 blocks as shown in Figure 2. Associated with each 2-Kbyte cache are 128 21-bit tags.

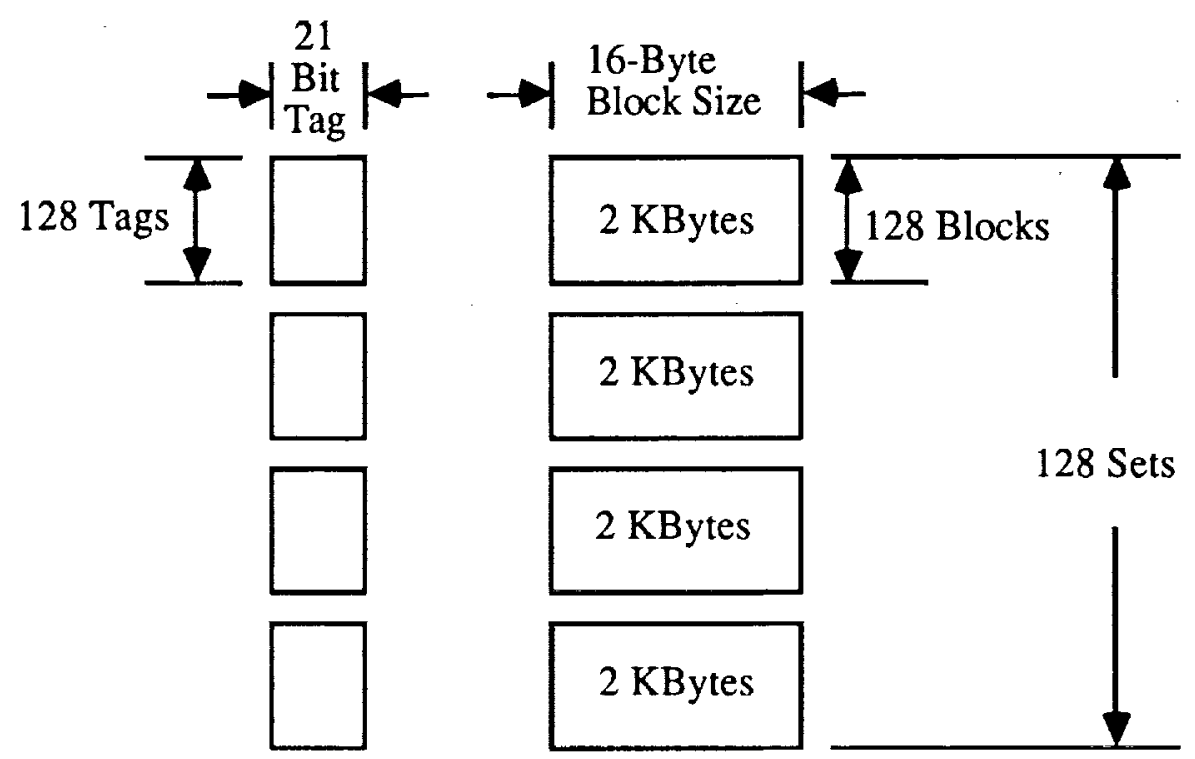

FIGURE 2. The i 486 On-Chip Cache Organization

The i486 on-chip cache, however, has neither parity check nor error detection and correction circuitry. When the cache is exposed in a high radiation environment, such as the Space Station Freedom, for a long period of time, a single event upset may occur, i.e. the contents of the cache memory may be altered. For this reason, it is necessary to measure the performance of the i486 with the cache disabled.

\subsection{The i486 On-Chip Cache Controlling Mechanism}

The i486 has four 32-bit control registers (CR0, 1, 2 and 3). Bit 30 (CD bit) and bit 29 (NW bit) in CR0 provide the on-chip cache control. The $\mathrm{CD}$ bit enables and disables the cache. The 
NW bit controls memory write-through and invalidates. The CD and NW bits define four operating modes of the cache (ref. 3), which are listed in Table 2.

TABLE 2. The i 486 on-chip cache operating modes

\begin{tabular}{|c|c|l|}
\hline CD & NW & \multicolumn{1}{|c|}{ Cache Operating Mode } \\
\hline 1 & 1 & Cache fills disabled, write-through and invalidates disabled \\
1 & 0 & Cache fills disabled, write-through and invalidates enabled \\
0 & 1 & INVALID. A fault with error code of 0 is raised \\
0 & 0 & Cache fills enabled, write-through and invalidates enabled \\
\hline
\end{tabular}

When the $C D$ and $N W$ bits are cleared $(C D=0$ and $N W=0)$, the cache is in the normal operating mode. The cache fills, write-through, and invalidates are enabled. The cache can be completely disabled by setting $\mathrm{CD}=1$ and $\mathrm{NW}=1$ and then flushing the cache. If the cache is not flushed, cache hits on reads will still occur and data will be read from the cache.

\subsection{The i486 Protection and Privilege Levels}

To disable the 1486 on-chip cache, the $C D$ and NW bits in CRO have to be set $C D=1$ and $\mathrm{NW}=1$ ). However, the $\mathrm{i} 486$ has four levels of protection to support the needs of a multi-tasking operating system. The four levels of protection are implemented by using four privilege levels (PLs) numbered 0 through 3 . Level 0 is the most privileged or trusted level and is used by the most essential routines (the operating system kernel). Application programs can operate only at the least privileged level, level 3 (Fig. 3.)

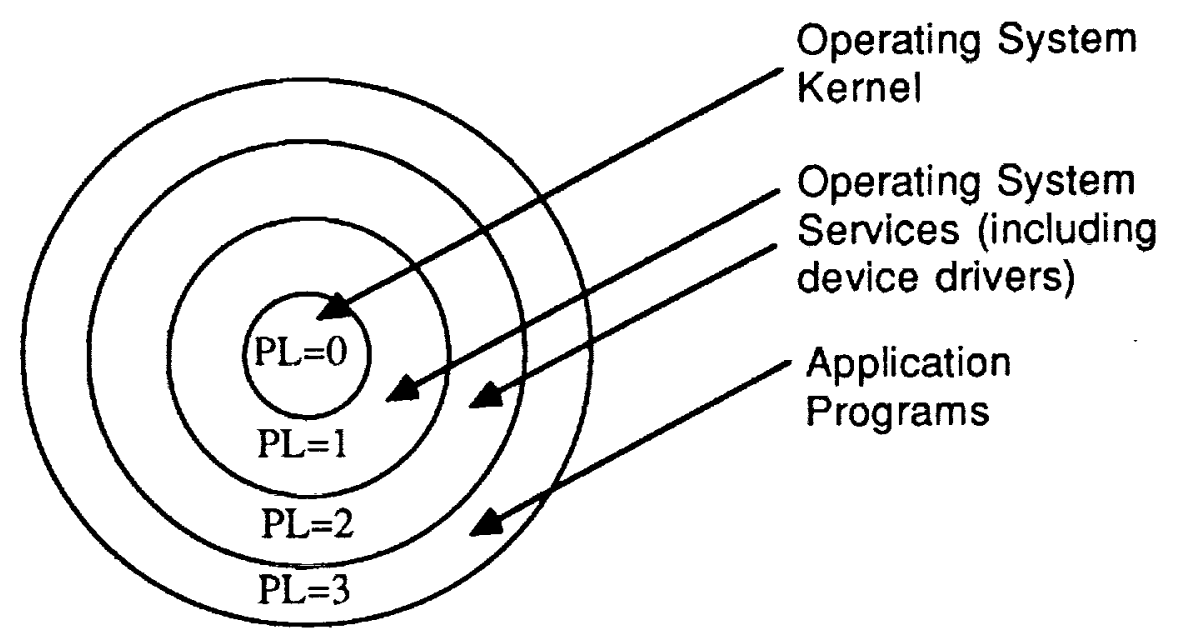

FIGURE 3. The i486 privilege levels

Privilege levels are used to improve the reliability of operating systems. By giving the operating system kernel the highest privilege, it is protected from damage by errors in other 
programs. If an application program crashes, the operating system has a chance to generate a diagnostic message and attempt to recover.

The privilege level determines which instructions from the instruction set can be executed by a task. Instructions that modify the system registers, such as CR0, are considered privileged instructions and can be executed only at privilege level 0 . Thus, the system registers can be modified only by the operating system kernel, never by application programs.

\subsection{Device Driver to Disable/Enable the i486 On-Chip Cache}

Device drivers provide interfaces between an operating system kernel and physical hardware devices. A device driver has the detail information of a particular device and hides these details from the operating system kernel. Device drivers are linked with the kernel and become part of the operating system, such as the Lynx real-time operating system shown in Figure 4. Most of the code in the LynxOS is implemented in C language, but many device drivers in the LynxOS have embedded assembly programs. The Lynx Assembler is used to assemble these assembly programs.

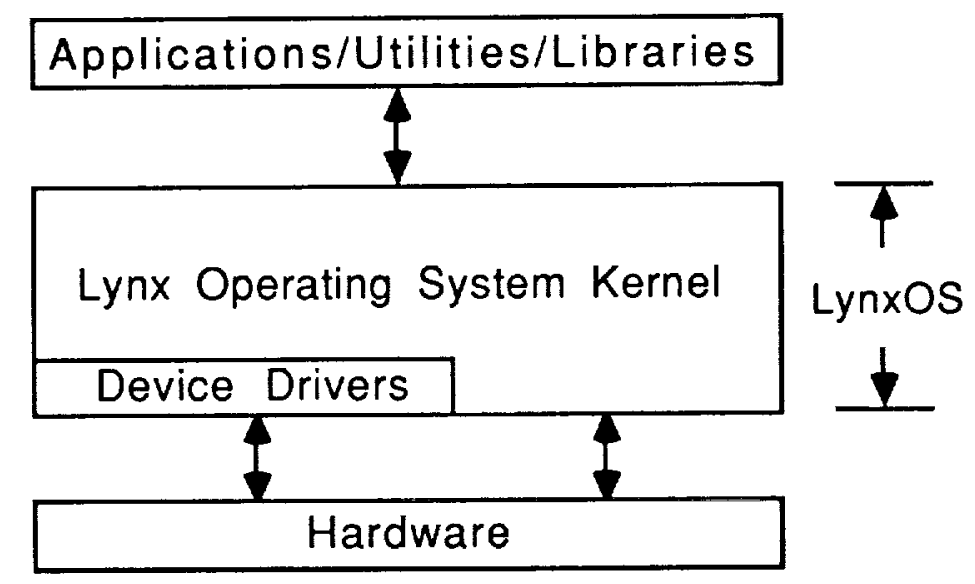

FIGURE 4. Lynx operating system organization

To disable/enable the i486 on-chip cache, a device driver was implemented; the complete program is listed in Appendix 7.1. The main algorithm of the program includes these steps:

- load the contents of CRO to a general-purpose 32-bit EAX register;

- set bit 29 and 30 of the EAX register to 1 for disabling the cache, or clear the two bits to 0 for enabling the cache;

- store the contents of EAX back to CRO; and

- flush the cache.

The implementation of the main algorithm to disable the i 486 on-chip cache is listed in Table 3. 
TABLE 3. Main algorithm of the device driver to disable the $i 486$ on-chip cache

\begin{tabular}{|ll|}
\hline mov & EAX, CRO \\
or & EAX, CRO_CD / CRO_NW \\
mov & CRO, EAX \\
invd & \\
\hline
\end{tabular}

\subsection{Errors Found in the Lynx Assembler}

Two errors were found in the Lynx Assembler (version 1.2) when implementing the device driver to disable/enable the 4886 on-chip cache: in storing the content of CR0 to the EAX register and in loading the contents of EAX to CR0. The main algorithm shown in Table 3 was assembled incorrectly by the Lynx Assembler and the results are shown in Table 4.

TABLE 4. The incorrect results of the main algorithm assembled by the Lynx Assembler

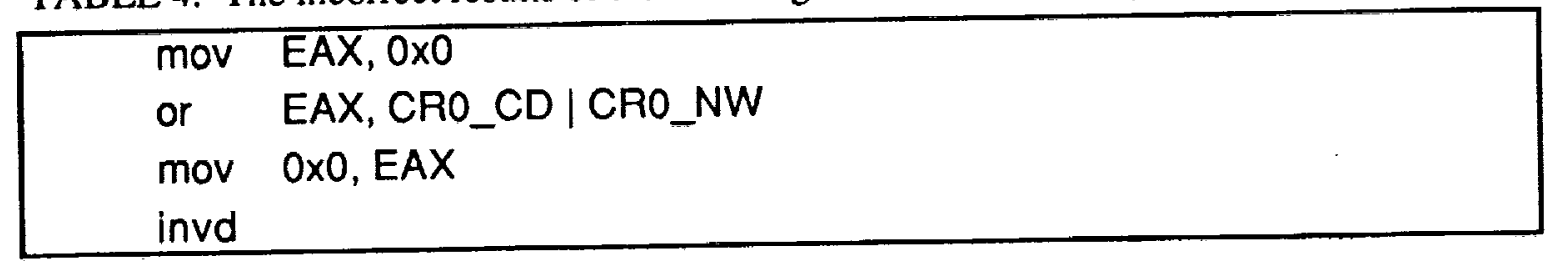

The instruction "mov EAX, CR0" was used to load the contents of CR0 to EAX. Instead of loading the contents of CRO to EAX, the Lynx Assembler actually assembled the instruction as "mov EAX, 0x0" and loaded 0 (zero) to EAX. When the instruction was executed, there was no warning or other message indicating the error.

The instruction "mov CR0, EAX" was used to store the contents of EAX back to CR0. Instead of storing the contents of EAX to CR0, it actually assembled the instruction as "mov $0 \mathrm{x} 0$, EAX" and stored the contents of EAX to address 0 (zero). Again, there was no warning or other message indicating the error when assembled. When the instruction was executed, however, the computer shut down ${ }^{\theta}$.

\subsection{Work Around the Problems}

The instructions of a microprocessor come from the instruction set and cause the microprocessor to execute an operation such as "MOV", "ADD" or "POP". They are translated by the assembler into machine language and are standardized across all assemblers. The instructions are often called "opcodes".

- The above errors were reported to Lynx Real-Time Systems, Inc. in October '90. Lynx RTS has recognized the problems and will correct them when they deliver the next version of the LynxOS to NASA Johnson Space Center (JSC) and IBM Federal Sector Division (FSD) in Houston, Texas. 
The directives of an assembler are not provided by the microprocessor instruction set. They are provided by individual assembler vendors, such as the Microsoft Assembler and the Lynx Assembler, and can vary from vendor to vendor. The directives are not translated into machine language. Instead, they provide instructions to the assembler itself. The directives are often called "pseudo-ops" to distinguish them from true opcodes (ref. 5).

The hexadecimal codes of the two "mov" instructions are listed in Table 5. The Lynx Assembler version 1.2 is a 386 -based assembler and does not support the i486. However, the "invd" instruction is one of the six new instructions being added to the i486 ISA and has to be used in the device driver to flush the 1486 on-chip cache. Therefore, this new instruction was not recognized by the Lynx Assembler version 1.2. The hexadecimal codes of this instruction are also listed in Table 5.

TABLE 5. Three instructions and its hexadecimal codes

\begin{tabular}{|l|l|}
\hline Instructions & Hexadecimal Codes \\
\hline mov EAX, CR0 & OF 20 C0 \\
\hline mov CR0, EAX & OF 22 C0 \\
\hline invd & OF 08 \\
\hline
\end{tabular}

To work around the problems mentioned in Section 4.5, an assembler directive "db" (define bytes) was used to replace the "mov" and "invd" instructions in the device driver as shown in Table 6.

TABLE 6. Using the "db" directive for the three instructions

\begin{tabular}{|lll|}
\hline $\mathrm{db}$ & OxOF, Ox20, OxCO & ;mov EAX, CRO \\
or & EAX, CRO_CD ICRO_NW & \\
$\mathrm{db}$ & OxOF, Ox22, OxCO & ;mov CRO, EAX \\
$\mathrm{db}$ & $0 \times 0 \mathrm{~F}, 0 \times 08$ & ;invd \\
\hline
\end{tabular}

\subsection{Application Programs to Interface with the Device Driver}

The device driver to disable and/or enable the 1486 on-chip cache was implemented, compiled, linked, and installed to make a new LynxOS. An application program is needed in order to interface with the device driver for disabling and/or enabling the cache. The application program for disabling the cache is shown in Appendix 7.2. The application program for enabling the cache is not shown because the only difference from the application program for disabling cache is that "OxAFFA" is replaced by "OxBFFB".

To verify the results of disabling/enabling the $\mathrm{i} 486$ cache, a routine named "kkprintf" (for kernel printing) was used in the device driver. Kkprintf is a printing mechanism provided for debugging a device driver. It sends all output to a fixed device, such as a terminal. 
A VT terminal was connected through a serial port in the PS/2 computer to verify the contents of CR0 after disabling and enabling the cache. When the application program to disable the $\mathrm{i} 486$ on-chip cache was executed, the message from "kkprintf" was displayed on the VT terminal as shown in Table 7.

TABLE 7. Display message for disabling the i486 on-chip cache Before disabling, CRO $=8000001 \mathrm{~b}$

After disabling, $\mathrm{CRO}=\mathrm{e} 000001 \mathrm{~b}$

When the application program to enable the cache was executed, the message was displayed on the VT terminal as shown in Table 8.

TABLE 8. Display message for enabling the i486 on-chip cache Before enabling, CRO $=\mathrm{e000001 \textrm {b }}$ After enabling, CRO $=8000001 \mathrm{~b}$ 


\section{COMPARISONS OF INTEL 386 AND i486 MICROPROCESSORS}

\subsection{Comparison of Instruction Set Architecture}

The i486 ISA (Instruction Set Architecture) has 229 instructions and is a super set of the 386 and 387 DX ISA. The i486 ISA has six more instructions than the 386/387 ISA: three for cache support (INVLPG, INVD, and WBINVD) and three for multiprocessing functions (CMPXCHG, XADD, and BSWAP) as shown in Table 9 . The 1486 is $100 \%$ binarily upward compatible with the $386 / 387$ because of the super set ISA. No modification, recompilation, or relinkage was needed for any of the software used in this analysis, including LynxOS, when the 386/387 DX and the $\mathrm{i} 486$ boards were swapped.

TABLE 9. The six new i486 instructions.

\begin{tabular}{|l|l|}
\hline \multicolumn{1}{|c|}{ Instructions } & \multicolumn{1}{c|}{ Functions } \\
\hline INVLPG & Invalidate TLB (translation-lookaside buffer) entry \\
\hline INVD & Invalidate cache \\
\hline WBINVD & Write-back and invalidate cache \\
\hline CMPXCHG & Compare and exchange \\
\hline XADD & Exchange and add \\
\hline BSWAP & Byte swap \\
\hline
\end{tabular}

\subsection{Comparison of Power Consumption}

As mentioned before, the 1486 contains both the integer unit and the floating-point unit. The power dissipation data for the $386 \mathrm{DX}, 387 \mathrm{DX}$, and i486, calculated from the power supply current (ref. 1, 3), are listed in Table 10 . The $25-\mathrm{MHz}$ i486 power dissipation $(3.5 \mathrm{~W}$ ) is lower than the sum of the $386 \mathrm{DX}$ and $387 \mathrm{DX}(4.8 \mathrm{~W})$. Therefore, the strict power consumption requirement for the SSF DMS does not cause a problem using the i 486 rather than the $386 / 387$ DX.

TABLE 10. The Power dissipation of the $386 / 387$ DX and i 486

\begin{tabular}{|l|l|}
\hline \multicolumn{1}{|c|}{ Microprocessor } & \multicolumn{1}{c|}{ Power Dissipation } \\
\hline $386 \mathrm{DX}(20,25,33 \mathrm{MHz})$ & $2.5,2.8,2.8 \mathrm{~W}$ \\
\hline $387 \mathrm{DX}(20,25,33 \mathrm{MHz})$ & $1.5,2.0,2.0 \mathrm{~W}$ \\
\hline $\mathrm{i} 486(25,33 \mathrm{MHz})$ & $3.5,4.5 \mathrm{~W}$ \\
\hline
\end{tabular}




\subsection{Comparison of Space Qualification Schedule}

The 25-MHz $386 \mathrm{DX}$ and $387 \mathrm{DX}$ qualified for MIL-STD-883C (ref. 6) Class B (for military applications) in 1989. Intel plans to have the $25 \mathrm{MHz} 386 \mathrm{DX}$ and $387 \mathrm{DX}$ meet the Class S qualification (for space applications) 52 weeks after the order is placed.

Intel plans to have the $25-\mathrm{MHz}$ i 486 meet the Class B qualification (for military applications) in 1992, and Class S qualification at the end of 1993 (ref. 7).

\subsection{Performance Comparison}

The hardware and software configurations and the benchmark programs used in this performance comparison are described in Section 3.

The performance were measured with six configurations:

(1) i486 with 8-KB on-chip cache;

(2) 1486 with the 8-KB on-chip cache disabled;

(3) PS/2 Model 70 with 64-KB external cache;

(4) PS/2 Model 70 with the 64-KB external cache disabled;

(5) prototype EDP (Embedded Data Processor); and

(6) prototype FEU (Flight Equivalent Unit).

The performance results from the six configurations are listed in Table 11. The results indicate that:

1. The performance of the $i 486$ with the internal cache (Configuration 1) is about two to three times higher than the $386 / 387 \mathrm{DX}$ with external cache (Configuration 3).

2. The performance of the $i 486$ with the internal cache disabled (Configuration 2) still has higher floating-point performance than the 386/387 with or without external cache (Configuration 3 or 4) due to the on-chip floating-point unit.

3. The performance of the i 486 with the internal cache disabled (Configuration 2), however, has lower integer performance than the 386/387 with or without external cache (Configuration 3 or 4).

4. The configuration of the PS/2 Model 70 with cache disabled (Configuration 4) is similar to the prototype EDP (Configuration 5). The benchmark results are also close to each other.

5. The performance of the prototype FEU (Configuration 6 ) is only about $50 \%$ of the PS/2 Model 70 with cache (Configuration 3), which is generally considered as a 4 MIPS (million instructions per second) computer. 
TABLE 11. Summary of performance comparison

\begin{tabular}{|l|l|l|l|l|l|l|}
\hline $\begin{array}{l}\text { Configur- } \\
\text { ation No. }\end{array}$ & $\mathbf{1}$ & $\mathbf{3}$ & $\mathbf{3}$ & $\mathbf{4}$ & $\mathbf{5}$ & $\mathbf{6}$ \\
\hline Items & $\mathrm{i486}$ & $\mathrm{i486}$ & $\begin{array}{l}\text { PS/2 } \\
\text { Model 70 }\end{array}$ & $\begin{array}{l}\text { PS/2 } \\
\text { Model 70 }\end{array}$ & $\begin{array}{l}\text { Prototype } \\
\text { EDP }\end{array}$ & $\begin{array}{l}\text { Prototype } \\
\text { FE U }\end{array}$ \\
\hline $\begin{array}{l}\text { Micro- } \\
\text { processors }\end{array}$ & $\mathrm{i} 486$ & $\mathrm{i} 486$ & $\begin{array}{l}386 \text { and } \\
387 \mathrm{DX}\end{array}$ & $\begin{array}{l}386 \text { and } \\
387 \mathrm{DX}\end{array}$ & $\begin{array}{l}386 \text { and } \\
387 \mathrm{DX}\end{array}$ & $\begin{array}{l}386 \text { and } \\
387 \mathrm{DX}\end{array}$ \\
\hline Clock & $25 \mathrm{MHz}$ & $25 \mathrm{MHz}$ & $25 \mathrm{MHz}$ & $25 \mathrm{MHz}$ & $20 \mathrm{MHz}$ & $20 \mathrm{MHz}$ \\
\hline $\begin{array}{l}\text { External } \\
\text { cache }\end{array}$ & $\mathrm{N} / \mathrm{A}$ & $\mathrm{N} / \mathrm{A}$ & $64 \mathrm{~KB}$ & Disabled & N/A & N/A \\
\hline $\begin{array}{l}\text { Internal } \\
\text { cache }\end{array}$ & $8 \mathrm{~KB}$ & Disabled & N/A & N/A & N/A & N/A \\
\hline $\begin{array}{l}\text { Main } \\
\text { memory }\end{array}$ & $4 \mathrm{MB}$ & $4 \mathrm{MB}$ & $4 \mathrm{MB}$ & $4 \mathrm{MB}$ & $4 \mathrm{MB}$ & $4 \mathrm{MB}$ \\
\hline $\begin{array}{l}\text { Error } \\
\text { correction } \\
\text { codes }\end{array}$ & N/A & N/A & N/A & N/A & N/A & Yes \\
\hline $\begin{array}{l}\text { Single event } \\
\text { upset scrub }\end{array}$ & N/A & N/A & N/A & N/A & N/A & Yes \\
\hline $\begin{array}{l}\text { Operating } \\
\text { system }\end{array}$ & $\begin{array}{l}\text { LynxOS } \\
\text { (v 1.2) }\end{array}$ & $\begin{array}{l}\text { LynxOS } \\
(\mathrm{v} 1.2)\end{array}$ & $\begin{array}{l}\text { LynxOS } \\
(\mathrm{v} 1.2)\end{array}$ & $\begin{array}{l}\text { LynxOS } \\
\text { (v 1.2) }\end{array}$ & $\begin{array}{l}\text { LynxOS } \\
\text { (v 2.0) }\end{array}$ & $\begin{array}{l}\text { LynxOS } \\
\text { (v 2.0) }\end{array}$ \\
\hline $\begin{array}{l}\text { Compiler } \\
\text { Lynx C }\end{array}$ & Lynx C & Lynx C & Lynx C & Lynx C & Lynx C \\
\hline Dhrystone & 13680 & 2903 & 7196 & 3970 & 4307 & 3016 \\
\hline Whetstone & 4153 & 1539 & 1330 & 1084 & 912 & 858 \\
\hline
\end{tabular}




\section{CONCLUSIONS AND RECOMMENDATIONS}

The i486 demonstrates the following advantages over the $386 / 387 \mathrm{DX}$ :

(1) lower power consumption than the combination of the $386 \mathrm{DX}$ and $387 \mathrm{DX}$; and

(2) higher floating-point performance: even with the on-chip cache disabled, the i486 still has higher floating-point performance in speed than the $386 / 387 \mathrm{DX}$.

The i486 on-chip cache, however, has neither parity check nor error detection and correction circuitry. In space, the probability of having the contents of the cache altered, is higher than on the ground due to the higher radiation exposure. With the on-chip cache disabled, the 1486 fixed-point (integer) performance in speed was heavily penalized. Using external cache with a specially designed cache controller can improve the performance of the i486, but the added complexity in bus coherency may not provide a better solution than adding cache to the $386 / 387 \mathrm{DX}$ configuration. Besides, some compilers designed for the 1486 may be optimized by using the onchip cache. Using the $\mathrm{i} 486$ with the on-chip disabled may not benefit from these compilers.

The benchmark performance of a 386-based prototype Flight Equivalent Unit (FEU), which is the closet configuration to the DMS design as of April 1991, is only about 50\% of a PS/2 Model 70 with cache, which is generally considered as a 4 MIPS computer. Adding cache to the 386/387 DX memory hierarchy appears to be the most beneficial way to enhance computation-intensive performance for the current DMS design at this time. 


\section{APPENDIX: PROGRAMS AND EXAMPLES}

7.1. Listing of the Device Driver to Disable/Enable the i486 Cache

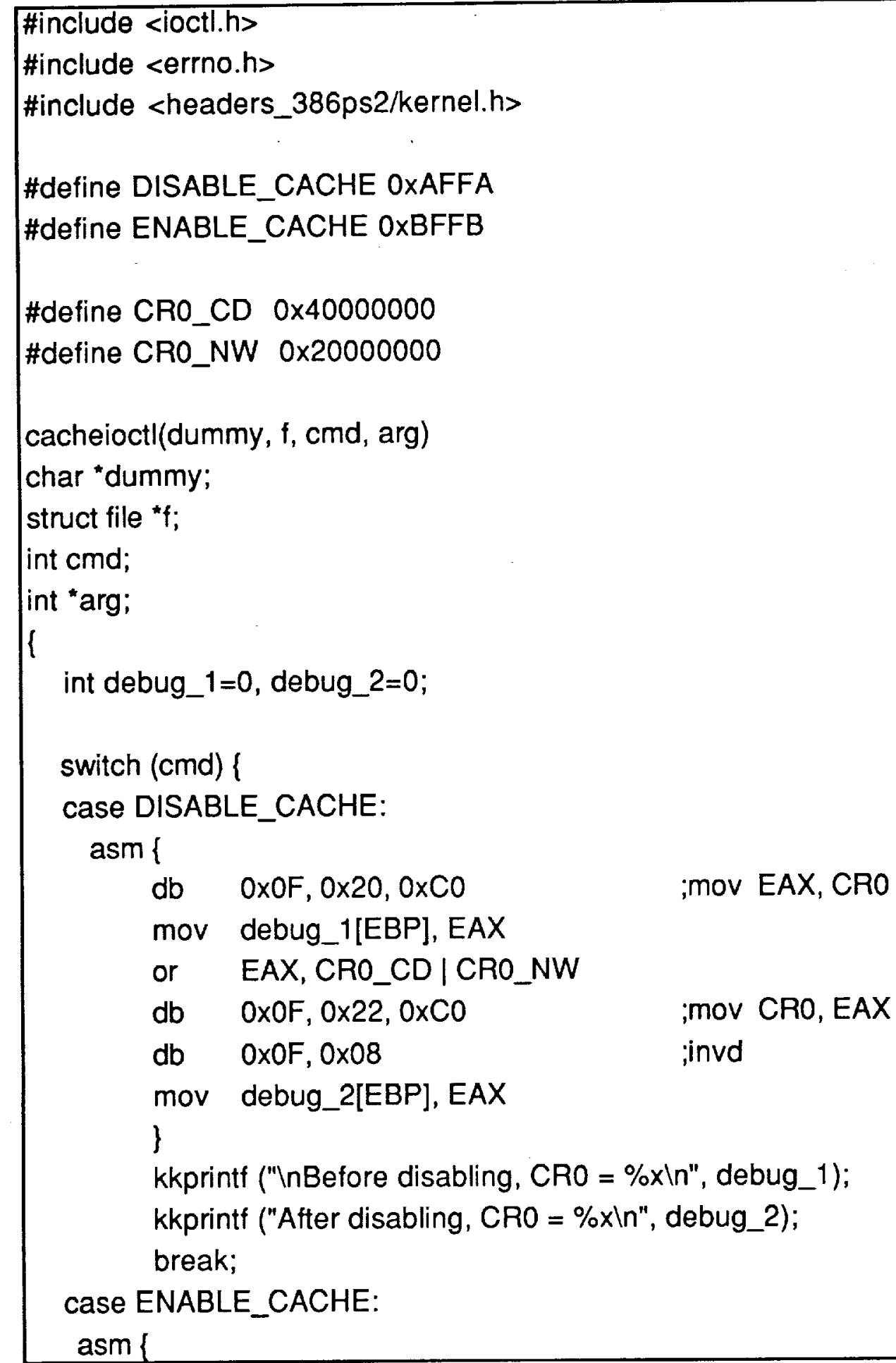




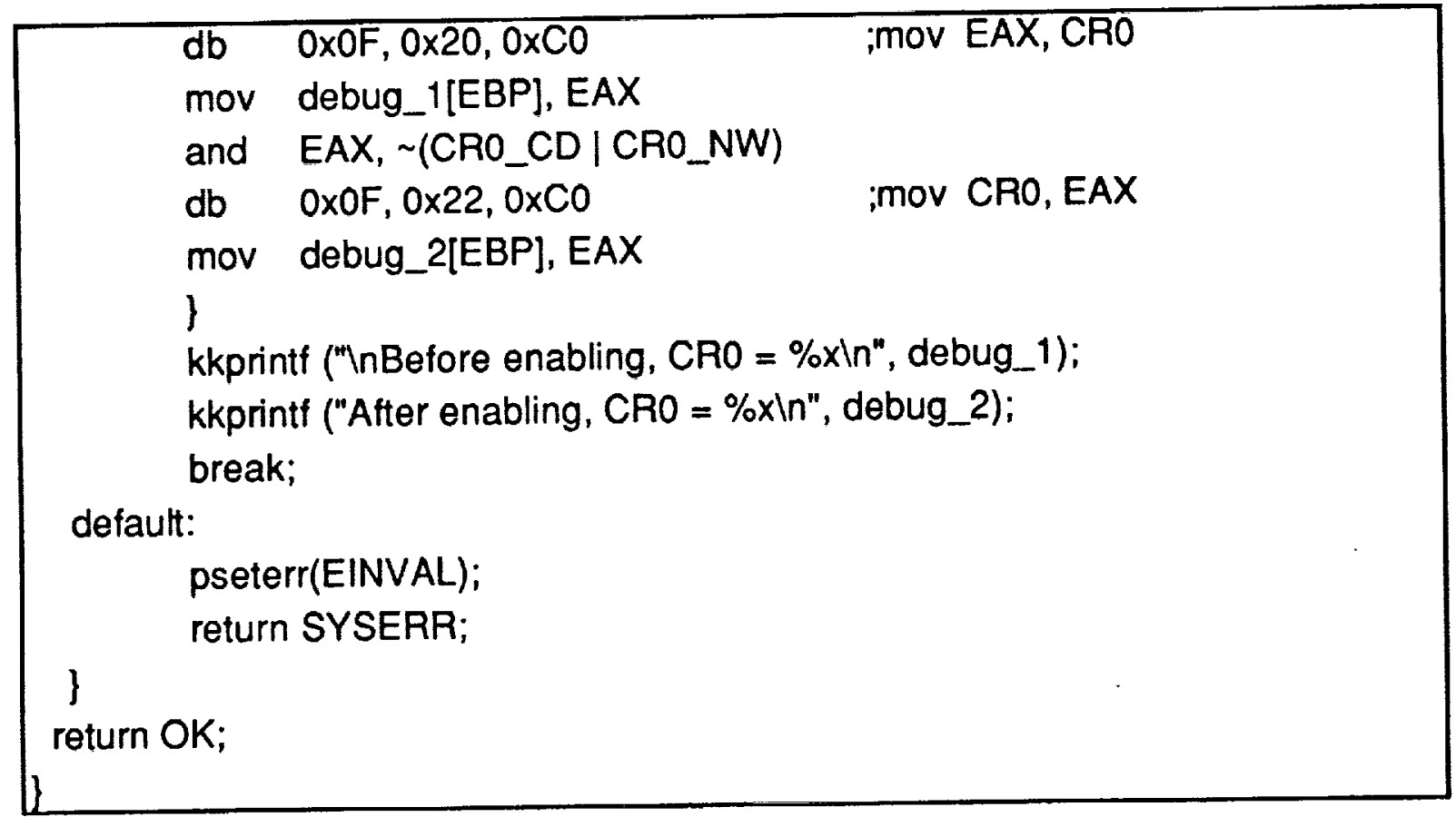

7.3. Listing of the Application Program to Disable the i486 Cache

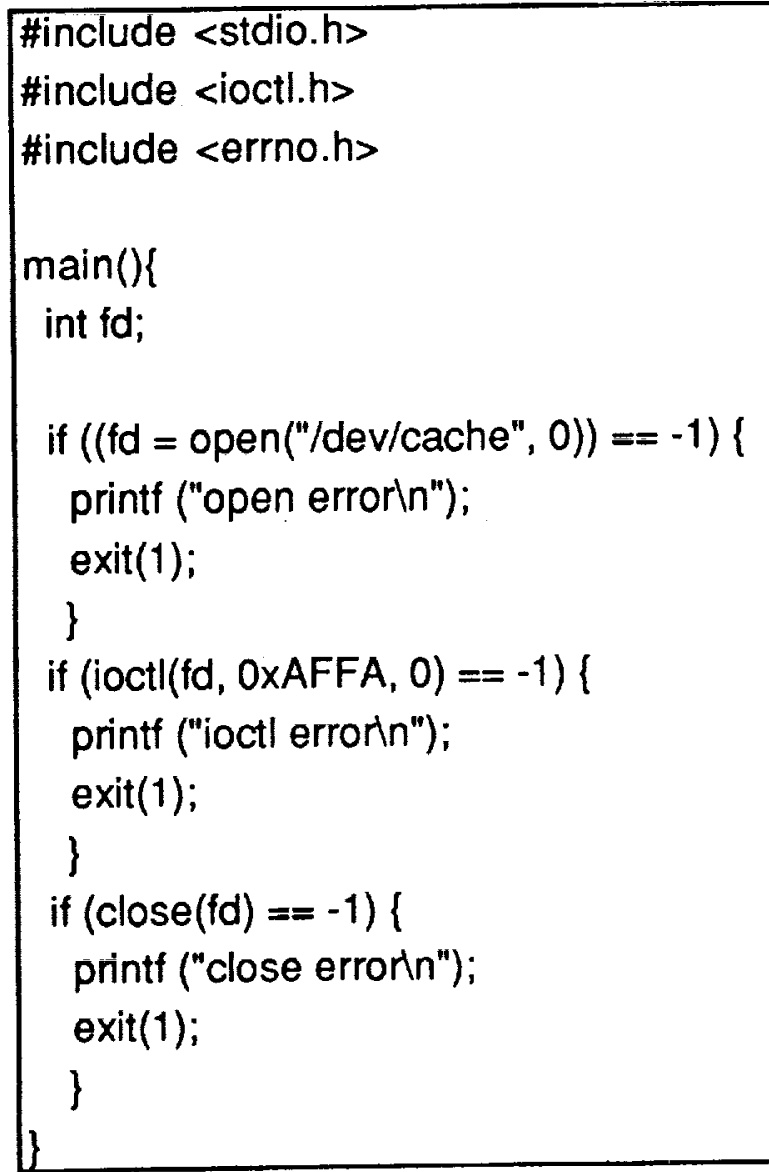




\subsection{Example of A Dhrystone Benchmark Result}

\begin{tabular}{|c|c|}
\hline \multicolumn{2}{|c|}{ Dhrystone Benchmark, Version 2.1 (Language: C) } \\
\hline \multicolumn{2}{|c|}{ Program compiled without 'register' attribute } \\
\hline \multirow{3}{*}{\multicolumn{2}{|c|}{$\begin{array}{l}\text { Please give the number of runs through the benchmark: } \\
\text { Execution starts, } 100000 \text { runs through Dhrystone } \\
\text { Execution ends }\end{array}$}} \\
\hline & \\
\hline & \\
\hline \multicolumn{2}{|c|}{ Final values of the variables used in the benchmark: } \\
\hline Int_Glob: & 5 \\
\hline should be: & 5 \\
\hline Bool_Glob: & 1 \\
\hline should be: & 1 \\
\hline Ch_1_Glob: & A \\
\hline should be: & A \\
\hline Ch_2_Glob: & B \\
\hline should be: & B \\
\hline Arr_1_Glob[8]: & 7 \\
\hline should be: 7 & 7 \\
\hline $\begin{array}{c}\text { Arr_2_Glob[8][7]: } 100 \\
\text { should be: }\end{array}$ & $\begin{array}{l}0010 \\
\text { Number_Of_Runs }+10\end{array}$ \\
\hline Ptr_Glob-> & \\
\hline $\begin{array}{l}\text { Ptr_Comp: } \\
\text { should be: }\end{array}$ & $\begin{array}{l}31804 \\
\text { (implementation-dependent) }\end{array}$ \\
\hline $\begin{array}{cc}\text { Discr: } & 0 \\
& \text { should be: }\end{array}$ & 0 \\
\hline $\begin{array}{c}\text { Enum_Comp: } 2 \\
\text { should be: }\end{array}$ & 2 \\
\hline Int_Comp: & 17 \\
\hline should be: & 17 \\
\hline $\begin{array}{l}\text { Str_Comp: } \\
\text { should be. }\end{array}$ & $\begin{array}{l}\text { DHRYSTONE PROGRAM, SOME STRING } \\
\text { DHRYSTONE PROGRAM SOME STRING }\end{array}$ \\
\hline Next_Ptr_Glob-> & \\
\hline Ptr_Comp: & 31804 \\
\hline
\end{tabular}




\begin{tabular}{|c|c|c|}
\hline \multicolumn{3}{|c|}{ should be: (implementation-dependent), same as above } \\
\hline \multicolumn{3}{|c|}{ Discr: $\quad 0$} \\
\hline shoulc & be: & 0 \\
\hline \multicolumn{3}{|c|}{ Enum_Comp: $\quad 1$} \\
\hline \multicolumn{3}{|c|}{ should be: 1} \\
\hline \multicolumn{2}{|c|}{ Int_Comp: } & 18 \\
\hline \multicolumn{2}{|c|}{ should be: } & 18 \\
\hline \multicolumn{2}{|c|}{ Str_Comp: } & DHRYSTONE PROGRAM, SOME STRING \\
\hline \multicolumn{2}{|c|}{ should be: } & DHRYSTONE PROGRAM, SOME STRING \\
\hline \multicolumn{2}{|c|}{ Int_1_Loc: } & 5 \\
\hline \multicolumn{2}{|c|}{ should be: } & 5 \\
\hline \multicolumn{2}{|c|}{ Int_2_Loc: } & 13 \\
\hline \multicolumn{2}{|c|}{ should be: } & 13 \\
\hline \multicolumn{2}{|c|}{ Int_3_Loc: } & 7 \\
\hline \multicolumn{2}{|c|}{ should be: } & 7 \\
\hline \multirow{2}{*}{\multicolumn{2}{|c|}{$\begin{array}{l}\text { Enum_Loc: } \\
\text { should be: }\end{array}$}} & 1 \\
\hline & & 1 \\
\hline \multirow{4}{*}{$\begin{array}{l}\text { Str_1_Loc: } \\
\text { should be: } \\
\text { Str_2_Loc: } \\
\text { should be: }\end{array}$} & & DHRYSTONE PROGRAM, 1'ST STRING \\
\hline & \multicolumn{2}{|c|}{ DHRYSTONE PROGRAM, 1'ST STRING } \\
\hline & & DHRYSTONE PROGRAM, 2'ND STRING \\
\hline & \multicolumn{2}{|c|}{ DHRYSTONE PROGRAM, 2'ND STRING } \\
\hline \multicolumn{3}{|c|}{ Microseconds for one run through Dhrystone: 73.2} \\
\hline
\end{tabular}

\subsection{Example of $A$ Whetstone Benchmark Result}

\begin{tabular}{|l|}
6.440 user time \\
0.030 system time \\
$0: 06.510$ elapse time \\
$99 \%$ cpu usage
\end{tabular}

(The derivation of the Whetstone performance in KWIPS is discussed in Section 3.3) 


\section{REFERENCES}

1. “386 DX Microprocessor Hardware Reference Manual," Intel Corp., Santa Clara, Calif., 1990.

2. “386 DX Programmer's Reference Manual," Intel Corp., Santa Clara, Calif., 1990.

3. "i486 Microprocessor," Intel Corp., Santa Clara, Calif., 1990.

4. "LynxOS User's Manual," Vol. 1, Version 1.2, Lynx Real-Time Systems, Inc., Los Gatos, Calif., May 1990.

5. Fernandez, N. Judi and Ashley, Ruth: “Assembly Language Programming For The 80386," McGraw-Hill, 1990, pp. 1-33.

6. "Military Standard Test Methods and Procedures for Microelectronics," U.S. Air Force, MILSTD-883C, 12 Feb. 1988.

7. Gregory L. Mather, Intel Corp., Chandler, Arizona, private communication, 1990. 


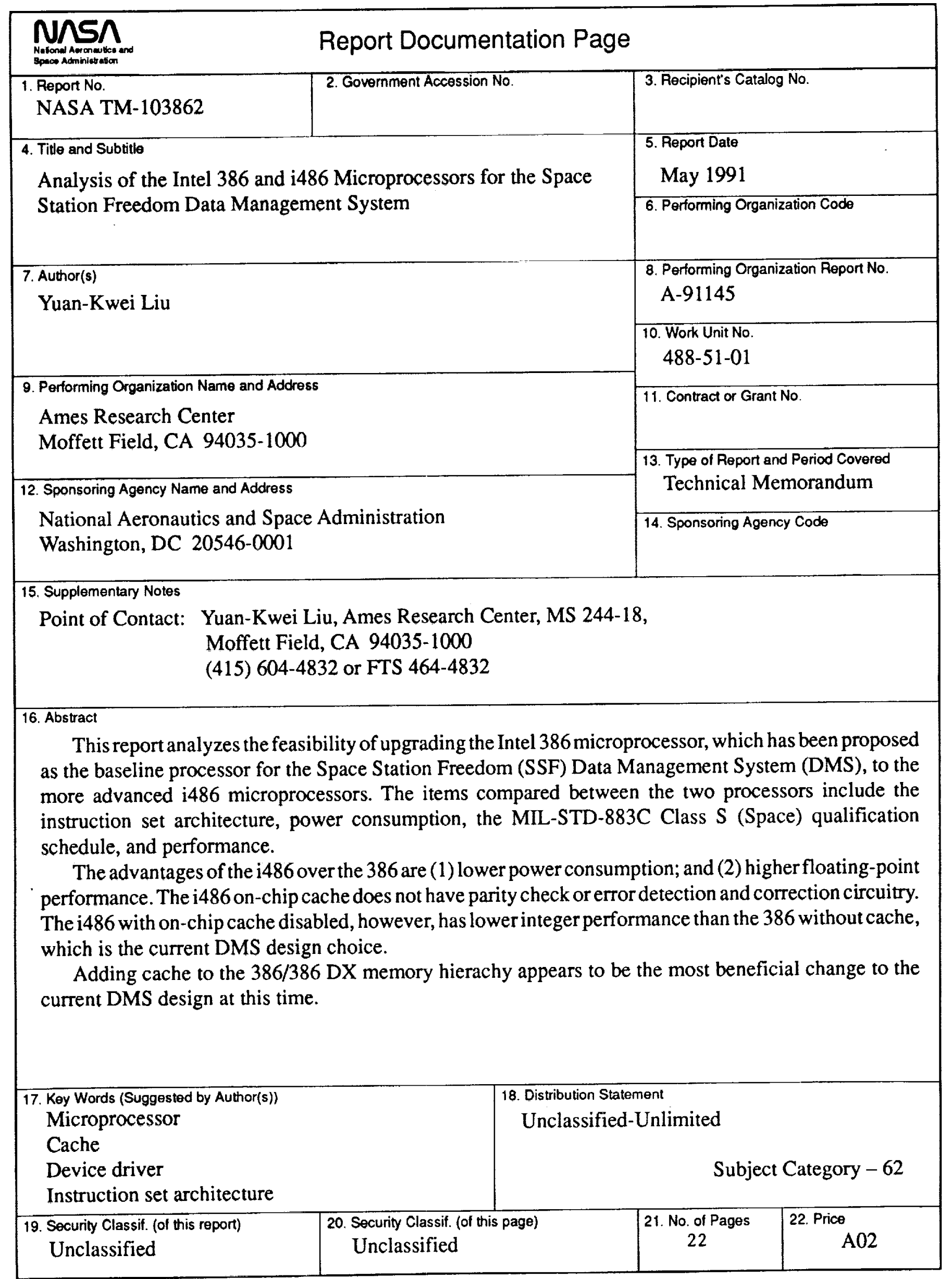

